\title{
Studies on an Electro-Hydraulic Load Input Tester for Fabrics
}

\author{
Part 1: Constructing the Load Input Tensile Tester \\ By Shigeru Aonuma and Takashi Murakami, Members, TMSJ \\ Faculty of Textile Science and Technology, Shinshu University, Ueda, Japan \\ Based on the Journal of the Textile Machinery Society of Japan, Vol. 27, No, 3, T35-45 (1974) \\ Abstract \\ The load input tensile tester, the performance of which is not influenced by the mesanical \\ properties of the material to be tested, was constructed. Its ability is investigated experiment- \\ ally, to show the following results. \\ 1) To avoid the velocity error the servo mechanism should satisfy the inequality:$$
1 / K_{s}+K_{p f} \ll K_{m} K_{f}
$$ \\ 2) To avoid the influence of the material properties to be tested, the hydraulic servo system \\ should satisfy the inequality: \\ $\left(A^{2} / k_{2}\right) K_{p f} \gg 1$ \\ 3) To make performance of the tester independent of the material properties to be tested, \\ a compensation circuit of the servo system should satisfy the conditions: \\ $K_{0} T_{2} T_{p p} \gg K_{p f} T_{1}$ \\ $G_{c}(s)=\left(T_{2} s+1\right) /\left(T_{1} s+1\right), \quad T_{2}>T_{1}$
}

Key WORDS: Tensile Testers, Fabric Property, Servomechanisms

\section{Introduction}

Although many studies ${ }^{[1-6]}$ have been done on the testing methods to know mechanical properties of woven fabrics, most of them belong to deformation inputtype and few to load input type, leaving only a touch of arbitrary load input type. However, in the actual textile production various loads act on fabrics and their deformation behavior is important to judge fabric characteristics. Woven fabrics are used not only as clothing materials but also as general industrial materials. But the load or deformation behavior of woven fabrics in practical use has not been discussed so much. The actual deformation input test of fabrics can be performed with a usual fabric tester. So we describe here a load input tester to measure the fabric deformation under such a load as simulating on actual production process on the hypothesis that the deformation characteristics of woven fabrics follow Voigt's model. The conditions to eliminate the influence of mechanical properties of tested materials on the characteristics of the tester are also discussed, and a few experimental results are described to show the abilities of this tester.

\section{Symbols Used}

The following symbols are used:

$f_{v}(t): \quad$ desired input load $(\mathrm{kg})$

90
$K_{1}$ : gain constant of the program generator to convert the input load into voltage $(\mathrm{V} / \mathrm{kg})$

$G_{c v}(s): \quad$ transfer function of the compensation circuit (1)

$G_{o}(s): \quad$ transfer function of the compensation circuit (2)

$K_{m}$ : gain constant of the hydraulic servo system $(\mathrm{cm} / \mathrm{V})$

$K_{d}$ : gain constant of the differential transfomer to convert the piston displacement into voltage $(\mathrm{V} / \mathrm{cm})$

$G_{h m}(s):$ transfer function of the hydraulic servo system

$x_{h}(t): \quad$ piston displacement in an actuator $(\mathrm{cm})$

$K_{s}$ : spring constant to convert displacement into load (extention force) $(\mathrm{kg} / \mathrm{cm}$ )

$f_{i}(t)$ : actual input load imposed on a test piece $(\mathrm{kg})$

$G_{p f}(s)$ : transfer function of the test piece under load input test.

Assuming Voigt's model, we put

$$
G_{p f}(s)=K_{p f} /\left(T_{p f} s+1\right)
$$

where

$$
T_{p f}=\lambda / \kappa, \quad K_{p f}=1 / \kappa
$$

$\lambda: \quad$ damper coefficient of the test piece $(\mathrm{kg} . \mathrm{sec} / \mathrm{cm})$ $\kappa: \quad$ spring constant of the test piece $(\mathrm{kg} / \mathrm{cm})$

$x_{o}(t)$ : deformation of the test piece under load input test $(\mathrm{cm})$

$K_{f}$ : gain constant of the converter to convert load Journal o The Textile Machinery Society of Japan 


\author{
into voltage $(\mathrm{V} / \mathrm{kg})$ \\ A: effective area of the cross section of the actuator \\ piston $\left(\mathrm{cm}^{2}\right)$ \\ $V: \quad$ volume of one side of the actuator (servo cylinder) \\ when the piston position is at the center of the \\ actuator $\left(\mathrm{cm}^{3}\right)$ \\ $k_{1}$ : characteristics of a spool valve $\left(\mathrm{cm}^{3} / \mathrm{sec} / X_{R}\right)$, \\ where $X_{R}$ is the spool displancement when rated \\ current $I_{R}$ is imposed on the servo valve. \\ $k_{2}$ : $\quad$ characteristics of a spool valve $\left(\mathrm{cm}^{5} / \mathrm{kg} \cdot \mathrm{sec}\right)$ \\ $\beta$ : compressive coefficient of the hydraulic oil \\ $\left(\mathrm{cm}^{2} / \mathrm{kg}\right)$ \\ $M$ : inertia of the load imposed on the hydraulic \\ servo system \\ $G_{e f}(s)$ : transfer function of a block diagram from the \\ desired input load to the actual load applied to \\ the the test piece \\ $K_{0}: \quad K_{\boldsymbol{o}}=\left|G_{c}(s)\right| K_{m} K_{\boldsymbol{f}}$ \\ $T_{1}, T_{2}$ : time constant of the compensation circuit
}

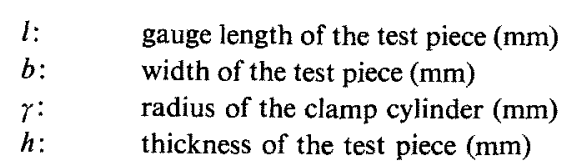

\section{Construction of the Tester}

This tester is composed of the following three parts: 1 ) a program generator which converts desired input load imposed on a test piece into input voltage according to a prescribed program function, 2) an input load controller which imposes load on a test piece in accurate proportion to input, 3) a detector and a recorder of fabric deformation.

Fig. 1 shows the construction of this tester. The electrohydraulic servo system is driven by the input voltage, and load is imposed on a test piece through the converter, fixed on the piston top of an actuator to convert the displacement into the actual load. The load applied to the test piece, after converted into voltage, is compared with the

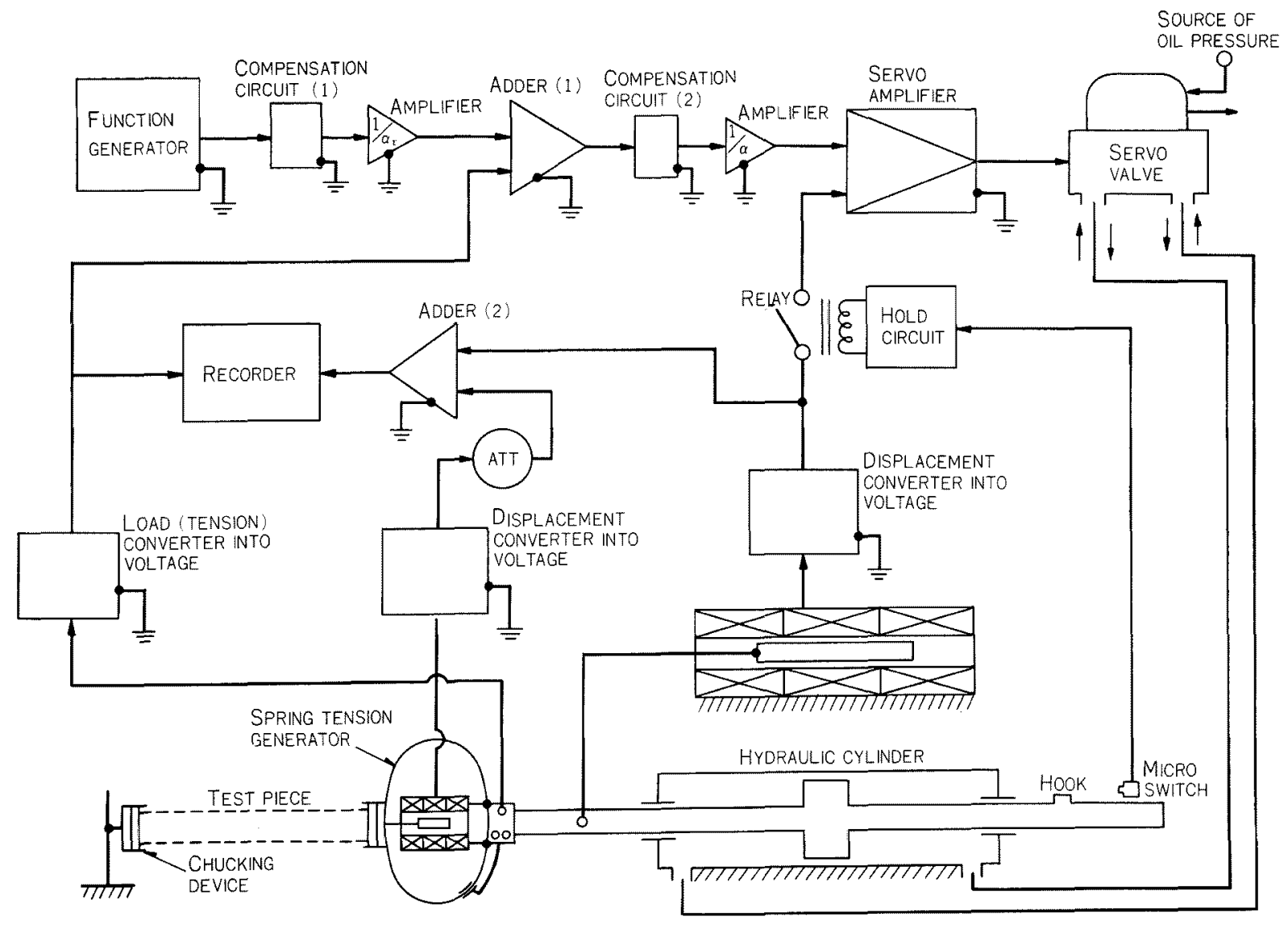

Fig. 1 Load input tensile tester for fabrics

Vol. 20 No. $4(1974)$ 
reference input voltage at the adder (1). The actuator piston is thus driven until the voltage difference becomes zero. So the input load can be imposed on a test piece accurately according to a prescribed program function.

On the other hand, the displacement of the test piece produced by load is the difference between the displacement of the piston and the deformation of the spring fixed at the piston top. In order to obtain this difference, the adder (2) is used to combine each voltage from two converters (in this care, differential transformers).

When a test piece is stretch-broken, the feed back loop of the input load controller is open. Therefore, a control circuit is necessary to return the piston back to the original position. For this purpose, when a hook on the piston rod touches a microswitch, a hold circuit and a negative feedback circuit are closed, the position is automatically returned to its original position.

Murakami already reported ${ }^{[7]}$ a load input tester, containing a local part in the servo system in order to improve the frequency characteristics of the tester, having such transfer function as the first order lag system. In the present article, a hydraulic servo system of an intergal element type is constructed in considering the steady-state deviation of the tester as described later.

Further, in order to compensate the loss of the frequency characteristics incured by the above construction, a compensation circuit (1) is set in the first part of the tester. A preamplifier (amplification factor is $1 / \alpha_{v}$ ) is used to compensate the gain loss caused by the insertion of phase lead compensation circuit, resulting in:

$$
\left|\boldsymbol{G}_{c v}(s)\right|=1
$$

The initial tension of a test piece is adjusted by adding suitable voltage to the adder (1). To start the test, a reference input voltage is imposed on the tester after the hold circuit is open.

Fig. 2 shows the hydraulic tensile apparatus, and Fig. 3 the input load controller and the recorder.

\section{Design of the Tester and Its Dynamic Characteristics}

The block diagram of this tester is shown in Fig. 4, in which the deformation of the test piece is added to the displacement of the actuator piston as feedback. This means $f_{i}(t)$ load imposed on a test piece, influenced by the mechanical properties of the test piece $G_{p f}(s)$. Murakami reported ${ }^{[8]}$ the means to solve this problem by inserting a phase lead compensation circuit. But when the steadystate deviation under ramp input is considered, an integral element should be contained in the transfer function of the hydraulic servo system, which therefore is constructed as follows:

$$
G_{h m}(s)=K_{m} / s
$$

Let us discuss the conditions to avoid the influence of

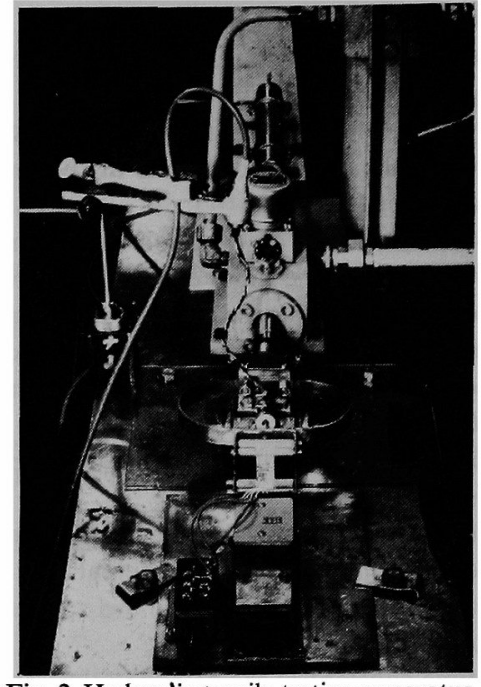

Fig. 2 Hydraulic tensile testing apparatus

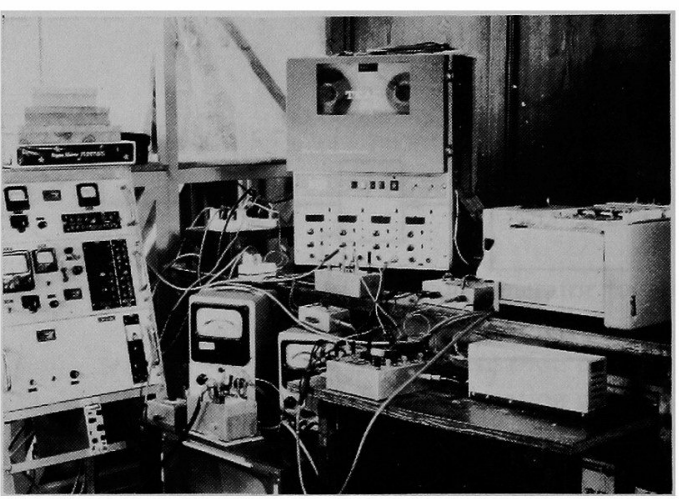

Fig. 3 Input load controller and recorde $r$

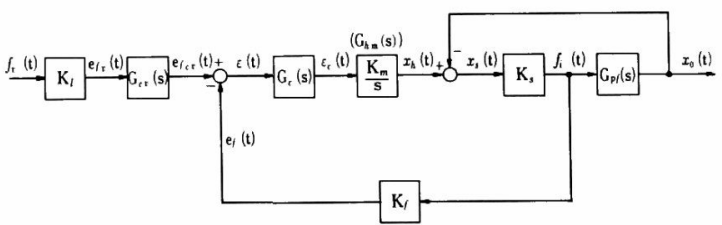

Fig. 4 Block diagram for load input tester

the properties of test materials and give a few examples of the tests.

4-1 Examination of the Steady-state Deviation

In Fig. 4, when the compensation circuit (2) $G_{c}(s)$ is not inserted in the system, viz. $G_{c}(s)=1$, the open-loop transfer function $G_{o}(s)$ is:

$$
G_{0}(s)=\frac{L\left\{e_{f}(t)\right\}}{L\{\varepsilon(t)\}}=\frac{K_{m} K_{s} K_{f}}{s\left\{1+K_{s} G_{p f}(s)\right\}}
$$


Several models are proposed to express the mechanical properties of woven fabrics, such as Voigt-Maxwell's fourelement model which includes a sliding block ${ }^{[9]}$. But in this paper, we assume that the test piece under load input follows Voigt's model ${ }^{[10]}$, and put.

$$
G_{p f}(s)=K_{p f} /\left(T_{p f} s+1\right) \text {. }
$$

From eq. (2), the steady-state deviation for a step input is :

$$
\varepsilon=\lim _{s \rightarrow 0}\left\{\frac{s\left(T_{p f s}+K_{s} K_{p f}+1\right)}{T_{p f s^{2}}+\left(K_{m} K_{s} K_{f} T_{p f}+K_{s} K_{p f}+1\right) s+K_{m} K_{s} K_{f}}\right\}
$$

However, when the compensation circuit (2) $G_{c}(s)$ is inserted as shown in Fig. 4, the steady-state deviation of this tester is zero, while the steady-state deviation for the ramp input (velocity error) settles down as follows:

$$
\varepsilon_{v}=\frac{1+K_{s} K_{p f}}{K_{m} K_{s} K_{f}}
$$

Thus so far as the hydraulic servo system is used, the steady-state deviation for the ramp input cannot be avoided. But, from eq. (4) if the following inequality is satisfied, the velocity error becomes negligible.

$$
1 / K_{s}+K_{p f} \ll K_{m} K_{f}
$$

For this purpose, it is necessary to increase the gain constants $K_{s}$ and $K_{m}$ and to raise the gain constant $K_{f}$ to an allowable limit. But as discussed in (4.4.E), if $K_{f}$ is increased too much, the hunting of the hydraulic servo system is induced. On the other hand, in order to satisfy the characteristics of the tester as a load input tester, the value of $K_{s}$ can not be increased extremely ${ }^{[11]}$. Accordingly, it is desirable to increase the gain constant $K_{s}$ and $K_{f}$ to an allowable limit together with raising the gain constant of $K_{m}$.

On the other hand, from eq. (5) only such test pieces can be applicable as having small $K_{p f}$, viz. large spring constant $\kappa$. So a question remains on stretchable materials such as bias-cut fabrics. However, as will be explained later, the above problem is solved by regulating the setting values of $K_{m}, K_{s}$ and $K_{f}$ and by inserting the compensation circuit into the system.

\section{4-2 Applicable Range of the Test Piece}

Generally the hydraulic servo system is subject to the influence of the material properties to be tested. But where the load on the hydraulic servo system is in a range applicable to the actuator, its influence is negligible. Since the transfer function of this tester has an integral element, the applicable range of the test piece will be discussed under this condition.

When the hydraulic servo system is loaded the relation between the spool displacement in the servo valve $x_{v}(t)$ and the displacement of the actuator piston $x_{h}(t)$ is expressed $^{[12]}$ as follows:

$$
\frac{L\left\{x_{h}(t)\right\}}{L\left\{x_{v}(t)\right\}}=\frac{k_{1} / A}{s+\left(1 / A^{2}\right)\left(k_{2}+\beta V / 2 \cdot s\right)(\lambda s+n)}
$$

In eq. (6), neglecting the compressibility of pressure oil, $\beta \div 0$

Accordingly, eq. (2) is:

$$
\frac{L\left\{x_{h}(t)\right\}}{L\left\{x_{v}(t)\right\}} \div \frac{A k_{1} /\left(A^{2}+k_{2} \lambda\right)}{s+k_{2} \lambda /\left(A^{2}+k_{2} \lambda\right)}
$$

Here, if the transfer function of the servo amplifier is shown by $K_{a}$, the transfer function of the hydraulic servo system is:

$$
G_{h}(s)=K_{a} \frac{L\left\{x_{h}(t)\right\}}{L\left\{x_{v}(t)\right\}}=\frac{K_{a} A k_{1} /\left(A^{2}+k_{2} \lambda\right)}{s+k_{2} \lambda /\left(A^{2}+k_{2} \lambda\right)}
$$

Therefore, so that the transfer function $G_{h}(s)$ may have an integral element as eq. (1), the following equation must be satisfied:

$$
\frac{1}{A^{2} / k_{2} \kappa+\lambda / \kappa}=0
$$

where, $\lambda / x$ is a time constant of the test piece, $T_{p f}$.

But as $T_{p f}$ measured with fabrics can not be found, $T_{p j}$ measured with fibers ${ }^{[13]}$ will be used. It is :

$$
T_{p f}=\lambda / \kappa=0.001 \sim 0.01(\mathrm{sec})
$$

While the value of $A^{2} / k_{2} k$ is large enough as will be shown later, so the following inequality can be satisfied:

$$
A^{2} / k_{2} \kappa=\left(A^{2} / k_{2}\right) K_{p j} \gg 1
$$

Therefore, the transfer function of the hydraulic servo system can be approximated to that having an integral element. To satisfy the condition of ineqality (10), it is desirable that the value of $k_{2}$ becomes small and the effective piston area becomes large. But it is undesirable to increase the effective area $A$, because the time constant of the hydraulic servo system is made large, Therefore, $k_{2}$ should be determind so as to make it applicable to a wide range of $K_{p j}$. The usual hydraulic servo system is designed in the following range:

$$
0.01<k_{2}<1 \quad\left(\mathrm{~cm}^{5} / \mathrm{kg} \cdot \mathrm{sec}\right)
$$

Therefore, when $A=4\left(\mathrm{~cm}^{2}\right), k_{2}=0.01$, the applicable range of $K_{p f}$ is:

$$
K_{p f}>0.0006(\mathrm{~cm} / \mathrm{kg}), \quad \text { Viz. } \pi<16666 \quad(\mathrm{~kg} / \mathrm{cm})
$$

Accordingly, it can be applicable to the test of fabrics with no problems.

\section{4-3 Insertion of the compensation circuit (2) $G_{c}(s)$}

Already Murakami reported ${ }^{[14]}$ the condition to avoid the influence of the properties of test materials by inserting a phase lead compensation circuit. In this paper, we also use the same technique, and so the imfluence by inserting the compensation circuit (2) will be discussed, As shown in the appendix, when the state equation of this system is transformed to a canonical form, this tester becomes uncontrollable and its observability may also break depending upon the condition of the tester. The tester is controllable on two variables, but uncontrollable on one variable. Accordingly, will be supposed the output trajectory of this system may be determined only with two variables. Also, when all the 
initial state values are zero, the load variable ( $y$ in the appendix) can be transfered from zero to the desired value. Although the characteristics equation of this tester are the third ordered, this system is stable. In Fig. 4, the transfer function $G_{c f}(s)$, in the range from desired load $f_{v}(t)$ to the actual load $f_{i}(t)$ imposed on the test piece, is:

$$
\begin{aligned}
& G_{c f}(s)=\frac{L\left\{f_{i}(t)\right\}}{L\left\{f_{v}(t)\right\}} \\
& =\frac{K_{1} K_{s} G_{c}(s) G_{h m}(s)}{1+K_{s}\left\{G_{p f}(s)+K_{f} G_{c}(s) G_{h m}(s)\right\}}
\end{aligned}
$$

The transfer function of the test piece $G_{p f}(s)$ is contained in the denominator of eq.(11). Therefore, it is necessary to design the compensation circuit which can neglect the characteristics of $G_{p f}(s)$. As $K_{f} G_{c}(s) G_{h m}(s)$ in the denominator of eq.(11) is a combination of three components contained in compensation circuit (2), a usual transfer function of compensation circuit is put into $G_{c}(s)$ :

$$
K_{f} G_{c}(s) G_{h m}(s)=\frac{K_{0}\left(T_{2} s+1\right)}{s\left(T_{1} s+1\right)}
$$

where

$$
K_{o}=\left|G_{c}(s)\right| K_{m} K_{f}
$$

In the denominator of eq. (11), inner \{\} is:

$$
\begin{aligned}
G_{p f}(s) & +K_{f} G_{c}(s) G_{h m}(s) \\
& =\frac{K_{p f}}{T_{p f} s+1}+\frac{K_{o}\left(T_{2} s+1\right)}{s\left(T_{1} s+1\right)} \\
& =\frac{\left(K_{0} T_{2} T_{p f}+K_{p f} T_{1}\right) s^{2}+\left\{K_{o}\left(T_{2}+T_{p f}\right)+K_{p f}\right\} s+K_{0}}{s\left(T_{p f} s+1\right)\left(T_{1} s+1\right)}
\end{aligned}
$$

In eq. (13), if the following inequality is satisfied,

$$
\begin{aligned}
& K_{0} T_{2} T_{p f} \gg K_{p f} T_{1} \\
& K_{o}\left(T_{2}+T_{p f}\right) \gg K_{p f}
\end{aligned}
$$

eq. (13) can be approximated as follows:

$$
G_{p f}(s)+K_{f} G_{c}(s) G_{h m}(s) \fallingdotseq \frac{K_{o}\left(T_{2} s+1\right)}{s\left(T_{1} s+1\right)}
$$

Further in eq. (14), as shown in (4-4-G):

$$
K_{o} T_{p f} \doteqdot K_{p f}
$$

So, the relation between $T_{1}$ and $T_{2}$ should satisfy:

$$
T_{2}>T_{1}
$$

Hence, $G_{c}(s)$ becomes the phase lead compensation circuit. Accordingly, to avoid the influence of the properties of the test piece, such phase lead compensation circuit (2) shonld be inserted as follows:

$$
G_{c}(s)=\left(T_{2} s+1\right) /\left(T_{1} s+1\right), \quad T_{2}>T_{1}
$$

The inequality eqs. (14) and (15) show that this tester is applicable to the case where the value of $K_{p f}$ is small. But this problem is actually out of the question by setting the condition of $K_{s}, K_{m}$ and $K_{f}$ as already descirbed.

From (4-1) and (4-3) described above, if eqs. (5), (10), (14) and (15) are satisfied, the influence of the properties of the test piece can be avoid, and accurate testing can be done.

\section{4-4 Design of Each Element}

The basic design of each element of this tester is as follows.

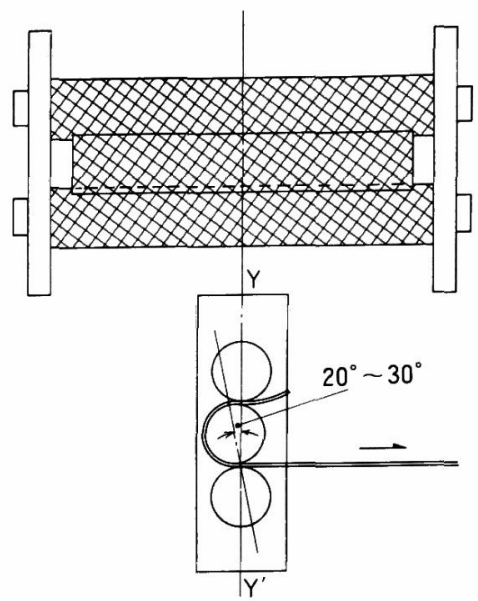

Fig. 5 Clamp part

\section{A. Test piece clamp}

As clamping of a test piece is important in testing fabrics, we used the combination of three knurled metallic cylinders as shown in Fig. 5. Two outer cylinders are fixed to the frame, leaving one cylinder free between them to roll a fabric around it. This clamp is a self-clamping mechanism and the clamping effect is inharced by the load imposed on a test piece.

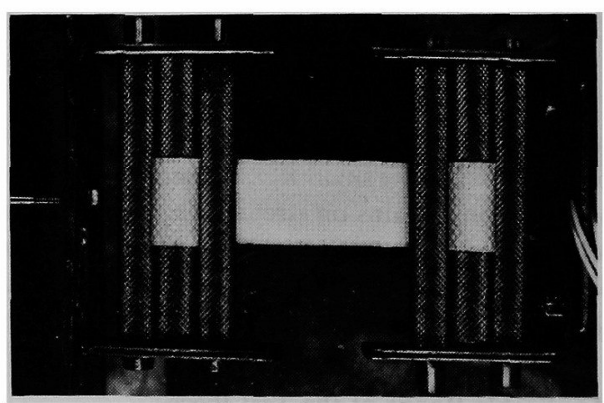

Fig. 6 Clams part

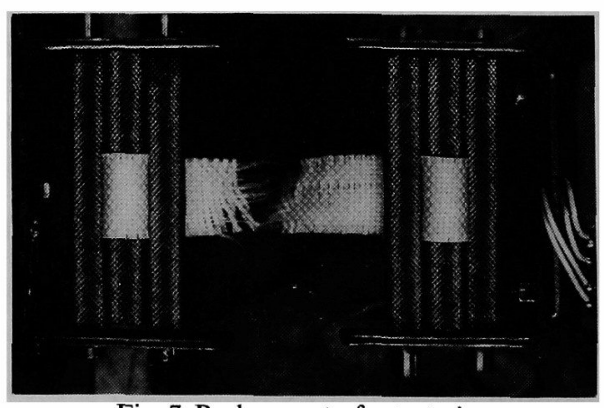

Fig. 7 Broken part of a test piece

Journal of The Textile Machinery Society of Japan 
Figs. 6 and 7 show the clamping ability which eliminates break down at the chucking line.

\section{B. Gauge length}

In Fig. 5, the gauge length of the test piece is between two contact lines of cylinders, $\overline{\mathbf{P P}^{\prime}}$ and $\overline{\mathbf{P P}^{\prime}}$. Accordingly the clamp position shifts by the thickness of the test piece.

Fig. 8 shows the enlarged drawing of the clamping part. $\mathrm{O}_{1}$ and $\mathrm{O}_{2}$ are the centers of outer fixed cylinders, $\mathrm{O}_{3}$ being the center of a movable cylinder when the test piece is not inserted, and $\angle \mathrm{O}_{2} \mathrm{O}_{1} \mathrm{O}_{3}=\theta$. If the test piece of thickness $h$ is inserted in the clamp center $\mathrm{O}_{3}$ shifts to $\mathrm{O}_{4}$ and contact line $\overline{\mathbf{P P}^{\prime}}$ shifts from $\mathrm{P}$ to $\mathrm{Q}$ along a locus vertical to $\overline{\mathrm{O}_{1} \mathrm{O}_{2}}$. The total shift length of the clamping line is,

$$
\left.\Delta l=2 \overline{\mathrm{PQ}}=2 \sqrt{(r+h / 2)^{2}-(r \cos \theta)^{2}}-r \sin \theta\right\}
$$

where, $r$ is the radius of metallic cylinders.

Table 1 shows the relation between the thickness $h$ and the shift length $A l$, where $r=4 \mathrm{~mm}$, and $\theta=20^{\circ}$.

Although the maximum stroke of the actuator is equal to $\pm 50 \mathrm{~mm}$ in length, the safety length is set at $\pm 40 \mathrm{~mm}$, and so the maximum stroke of the tester is $80 \mathrm{~mm}$. The breaking elongation of fabrics is generally about $20-30 \%$. But if the summation of deformation percentages of a test piece and of a spring which converts the displacement into the extention load is taken $160 \%$, the suitable gauge length is $50 \mathrm{~mm}$ in length. Easily extensible fabrics can be tested by using a short gauge length. From the above description, the basic dimensions of the clamp are as follows: $l=50 \mathrm{~mm}, r=4$ $\mathrm{mm}, \theta=20^{\circ}$.

The error of gauge length due to the thickness $h$ is within $5 \%$ for the practical use as shown in Table 1. For this reason, in order to set the gauge length correct, the thickness $h$ of a test piece should be measured beforehand.

C. Gain constant $\left(K_{m}\right)$ of hydraulic servo system

From eq. (5), it is desirable that the value of $K_{m}$ is large. Here, $K_{m}$ is a synthetical gain constant of the serveo amplifier, the servo valve and the actuator. Strictly speaking, the characteristics of these elements must be examined individually. But in this paper, the hydraulic servo system

Table 1. Relation between tickness $(h)$ and shift length of clamp position ( $\Delta l$ )

\begin{tabular}{ccc}
$h(\mathrm{~mm})$ & $\Delta l(\mathrm{~mm})$ & $(\Delta l / l) \times 100(\%)$ \\
\hline 0.10 & 0.28 & 0.56 \\
0.20 & 0.54 & 1.1 \\
0.30 & 0.78 & 1.6 \\
0.40 & 1.0 & 2.0 \\
0.50 & 1.2 & 2.5 \\
0.60 & 1.4 & 2.9 \\
0.70 & 1.6 & 3.3 \\
0.80 & 1.8 & 3.7 \\
0.90 & 2.0 & 4.0 \\
1.0 & 2.2 & 4.4 \\
\hline (where, $\gamma=4 \mathrm{~mm}$ & $\left.\theta=20^{\circ}, \quad l=50 \mathrm{~mm}\right)$
\end{tabular}

Vol. 20 No. 4 (1974)

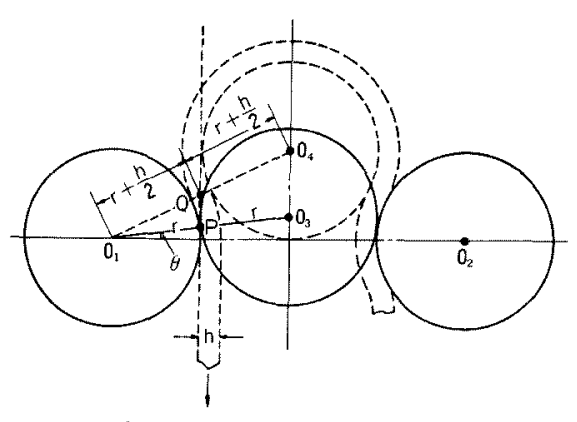

Fig. 8 Enlarged clamp part

Table 2 Gain characteristics of hydraulic servo system

\begin{tabular}{ccc}
\hline input voltage (V) & displacement $(\mathrm{cm})$ & $K_{m}(\mathrm{~cm} / \mathrm{V})$ \\
\hline 0.025 & 1.4 & 56 \\
0.050 & 4.4 & 88 \\
0.075 & 7.5 & 100 \\
0.10 & 10.0 & 100 \\
0.125 & 12.5 & 100 \\
0.15 & 13.8 & 92 \\
0.20 & 16.0 & 80 \\
0.30 & 18.5 & 62 \\
0.40 & 21.0 & 53 \\
0.50 & 24.0 & 48 \\
0.60 & 26.5 & 45 \\
0.70 & 29.3 & 42 \\
0.80 & 32.5 & 41 \\
0.90 & 33.0 & 37 \\
1.00 & 33.0 & 33 \\
\hline
\end{tabular}

Displacement: Displacement of piston by unit time to various step input voltage imposed hydraulic servo system

of this tester was designed based on the specification of each maker, and has the performance as follows:

$\begin{array}{lc}\text { rated flow } & 15(\mathrm{l} / \mathrm{min}) \\ \text { rated pressure } & 140\left(\mathrm{~kg} / \mathrm{cm}^{2}\right) \\ \text { band width at rated pressure } & 120(\mathrm{~Hz}) \\ \text { effective piston area } & 4\left(\mathrm{~cm}^{2}\right)\end{array}$

effective piston area $\quad 4\left(\mathrm{~cm}^{2}\right)$

Table 2 is an example of $K_{m}$ obtained experimentally from the relation between the characteristics of input and output of the hydraulic servo system. We used $K_{m} \fallingdotseq 100$ $\mathrm{cm} / \mathrm{V}$ in this tester. Rated flow of usual servo valves is from 15 to $30(l / \mathrm{min})$. If we want to increase $K_{m}$, it is possible to make to $K_{m}=1000(\mathrm{~cm} / \mathrm{V})$ when the rated flow is $180 \mathrm{l} / \mathrm{min}$.

D. Spring constant $K_{s}$

Although the value of $K_{s}$ is From eq. (5), desirable to be large, but it varies according to the experiment. In Fig. 4, as Murakami pointed out ${ }^{[15]}$, in order to satisfy the caracteristics of a load input tester, $K_{s}$ can not be increased so large due to the relation between the piston displacement in an actuator and the load which imposed to the 


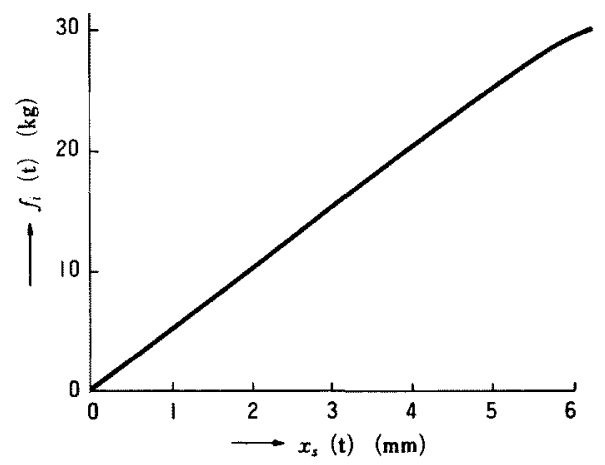

Fig. 9 Characteristics of spring converting displacement to load

test piece.

In this tester, the test pieces $15 \mathrm{~mm}$ by $50 \mathrm{~mm}$ in dimensions and having different $K_{s}$, are used. The preliminary experiment on general fabrics gave:

$K_{p f}=0.05-0.3(\mathrm{~kg} / \mathrm{cm})$

Breaking strength $=20 \mathrm{~kg}$

Considering these results, the spring constant $K_{s}$ which converts displacement into extension load was put to 50 $(\mathrm{kg} / \mathrm{cm})$. Fig. 9 shows the caracteristics of this spring. The relation between $x_{s}(t)$ and $f_{i}(t)$ is proportional within the region actually used as described in (4.4.E).

\section{E. Gain constant $\left(K_{f}\right)$}

From eq.(5), the value of $K_{f}$ is also desirable to be large. But as the maximum input voltage of the servo amplifier is $\pm 10 \mathrm{~V}$, it is desirable to set the magnitude of $K_{f}$ within $0.5(\mathrm{~V} / \mathrm{kg})$. In this tester, considering the hunting of the hydraulic servo system, $K_{f}$ was put to $0.2(\mathrm{~V} / \mathrm{kg})$.

F. Gain constant $K_{d}, K_{d o}$

As previously remarked, in order to get the deformaton output of a test piece as the relative displacement between the piston and the spring, the adder (2) is used to convert each displacement into voltage. Gain constants of these two converters are as follows.

Converter of the piston displacement to ele्riric voltage: measurement range $\pm 50(\mathrm{~mm}), K_{d}=0.13(\mathrm{~V} / \mathrm{mm})$.

Converter of the spring displacement to electric voltage: Measurement range $\pm 5(\mathrm{~mm}), K_{d o}=1.2(\mathrm{~V} / \mathrm{mm})$.

Accordingly, in order to balance both gains of converters a voltage attenuator was inserted in front of the adder (2).

G. Compensation circuit (2) $G_{c}(s)$

From eq. (14), $K_{o} T_{p f} T_{2} \gg K_{p f} T_{1}$

where $K_{\boldsymbol{o}}=\left|G_{c}(s)\right| K_{m} K_{f}$, and the gain of compensation circuit $\left|G_{c}(s)\right|$ is made equal to 1 by D.C amplifier.

By substituting $K_{m}=100(\mathrm{~cm} / \mathrm{V})$ and $K_{f}=0.2(\mathrm{~V} / \mathrm{kg})$ into the above equation, we have:

$$
K_{o} \doteqdot 20
$$

On the other hand, if we put

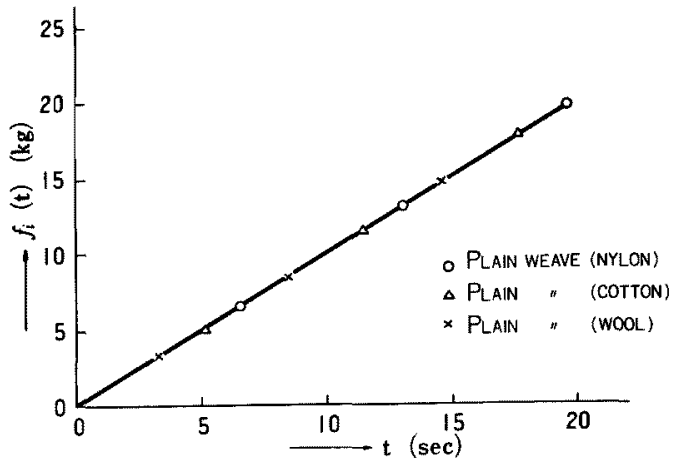

Fig. 10 Load $f_{i}(t)$ versus time tested under same ramp load

$T_{p f}=0.001-0.01(\mathrm{sec})$

$K_{p f}=0.05-0.3(\mathrm{~cm} / \mathrm{kg})$,

then $K_{o} T_{p f} \doteqdot K_{p f}$

Accordingly, in order to satisfy the inequality (14), the following inequality must be satisfied at least:

$$
T_{2}>T_{1} \text {. }
$$

So the compensation circuit becomes the type of phase lead. From eq. (12),

$$
G_{e}(s)=\frac{K_{o}}{K_{m} K_{f}} \cdot \frac{T_{2} s+1}{T_{1} s+1}=\frac{T_{2} s+1}{T_{1} s+1}
$$

In eq. (18), assuming $T_{2}$ to be the time constant of the servo system, using $T_{2}=0.02 \mathrm{sec}$ from Fig. 11, obtaining $T_{1}$ experimentally in order to set the velocity error to the minimum, the final design of $G_{c}(s)$ is as follows:

$$
G_{c}(s)=\frac{0.02 s+1}{0.01 s+1}=0.5 \frac{0.02 s+1}{0.5 \times 0.02 s+1}
$$

H. Compensation circuit (1) $G_{c v}(s)$

Because the transfer function of this hydraulic servo system has an integral element, the frequency response of this tester is not so good as that of the first order lag system containing a local part. Accordingly, in order to increase the band width of this tester, the phase lead compensation circuit has been inserted. As is clear from the Bode diagram in Figs. 11 and 12, the overall transfer function $G(s)$ of the

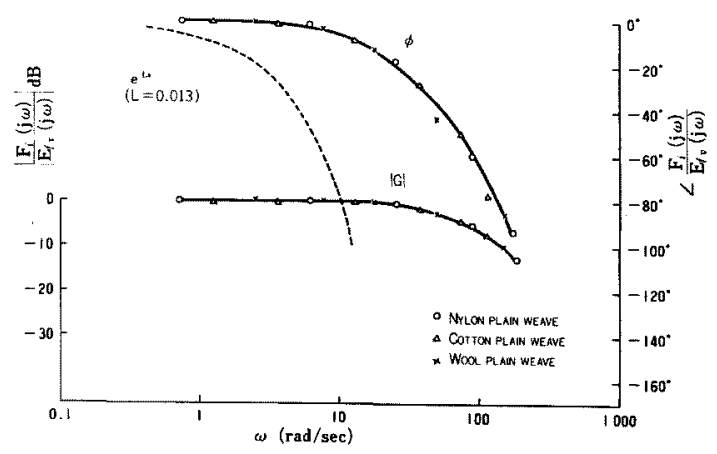

Fig. 11 Frequency response of load input tester

Journal of The Textile Machinery Society of Japan 


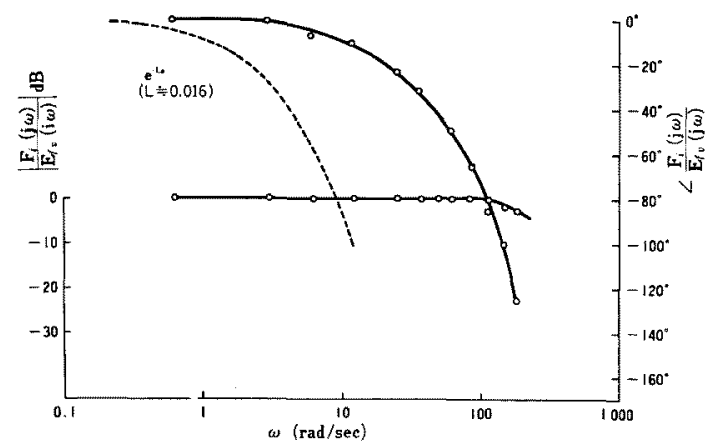

Fig. 12 Frequency response of load input tester when compensation circuit (1) is inserted

tester can be closely approximated to that of the system of the first order lag. Accordingly, we have:

$$
G(s)=\frac{K}{T s+1}
$$

Then in order to increase the band width of frequency characteristics the phase lead compensation circuit (1) $G_{c v}(s)$ is inserted in front of this tester as shown in Fig. 4. So,

$$
G_{c v}(s)=\alpha_{v} \frac{T_{c v} s+1}{\alpha_{v} T_{c v s} s+1}
$$

In eq. (20), $T_{c v}$ and $\alpha_{v}$ are determined to establish the following equation if $T_{c v}=T$.

$$
T_{e v}=0.02(\mathrm{sec}), \quad \alpha_{v}=0.23
$$$$
\text { where, }
$$$$
\alpha_{v} \frac{T_{\mathrm{cv} s+1}}{\alpha_{v} T_{c v s}+1} \cdot \frac{K}{T s+1} \doteqdot \alpha_{v} \frac{K}{\alpha_{v} T_{c v} s+1}
$$

From the Bode diagram in Fig. 11, we put $T=0.02(\mathrm{sec})$ and $\alpha_{v}=0.23$ to increase the band width of this tester. The gain drop caused by the insertion of the above compensation circuit is raised to 1 by using an amplifier having the amplification factor equal $1 / \alpha_{v}$.

\section{Performance and Experiment}

This tester was designed on the above description, and was examined its performance by changing $K_{p j}$ and by obtaining the transfer characteristics. Several experimental results obtained are as follows:

\subsection{Performance}

A. Velocity error (steady-state deviation for the ramp input)

It is necessary to examine the adequacy of the above conditions in order to decrease the velocity error by giving the hydraulic servo system an integral element and by designing so as to satisfy the conditions of eqs. (5), (10), (14) and (15). So load $f_{i}(t)$ was measured under the same ramp load input on various test pieces shown in Table 3,
Table 3 Spring constants of test pieces

\begin{tabular}{lc} 
kind of fabric* & $K_{p f}(\mathrm{~cm} / \mathrm{kg})$ \\
\hline Cotton plain weave & 0.095 \\
Wool plain weave & 0.12 \\
Nylon plain weave & 0.14 \\
\hline
\end{tabular}

(*length $l=50 \mathrm{~mm}$, width $b=15 \mathrm{~mm}$ )

Fig. 10 shows the results obtained, illustrating that the load $f_{i}(t)$ follows the load input signal faithfully within the tested region for various $K_{p f}$ and that the velocity error is completely avoided. As will be described in the after (5.2.A), the velocity error is also eliminated in wide range of $K_{p f}$, proving the success of this investigation.

B. Frequency response

As is explained before, the frequency characteristics of this tester are not so good as that of the type of the first order lag system ${ }^{[16]}$, namely, the frequency band width of this tester is $\omega=50 \mathrm{rad} / \mathrm{sec}$ while that of the first order lag type is $\omega=120 \mathrm{rad} / \mathrm{sec}$ (Fig. 11). The phase lag can be approximated to $e^{-L S}$ from the experimental results. So the decrease of band width has been compensated by using a phase lead compensation circuit. Fig. 12 shows the frequency band width when the compensation circuit (1) is used, in which

$$
T_{c v}=0.02(\mathrm{sec}), \alpha_{v}=0.23
$$

From Fig. 12, it is found that the band width of this compensated tester is improved to $\omega=210(\mathrm{rad} / \mathrm{sec})$, resulting in the maximum output amplitude of $10 \mathrm{~mm}$. In this case, as the phase lage can also be approximated to $e^{-L S}$, relatively simple composite wave form can be reproduced faithfully at least up to $\omega=120(\mathrm{rad} / \mathrm{sec})$ with the phase angle delayed by dead time alone.

\section{5-2 Experimental Results}

A. Deformation characteristics of bias cut fabrics.

Because the mechanical properties of fabrics are varied according to the bias angle of th fabric, the response for the ramp load input has been tested and compared. Here, it is necessary to prove experimentally that the characteristics of this tester are not subject to the influence of various bias: cut fabrics (Table 4). Fig. 13 shows the result of this test

Table 4 Spring constants of test pieces

\begin{tabular}{lc}
\hline bias cut angle* & $K_{p f}(\mathrm{~cm} / \mathrm{kg})$ \\
\hline $0^{\circ}$ (warp direction) & 0.046 \\
$5^{\circ}$ & 0.055 \\
$10^{\circ}$ & 0.015 \\
$15^{\circ}$ & 0.15 \\
$30^{\circ}$ & 0.23 \\
$45^{\circ}$ & 0.30 \\
\hline
\end{tabular}

*length of test piece $l=50 \mathrm{~mm}$

width of test piece $b=15 \mathrm{~mm}$

$* \frac{3}{3}$ basket weave 


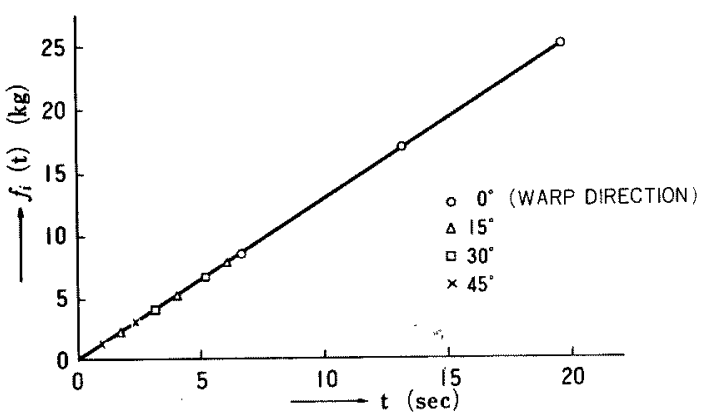

Fig. 13 Load $f_{i}(t)$ of different bias cut fabrics tested under same ramp load input

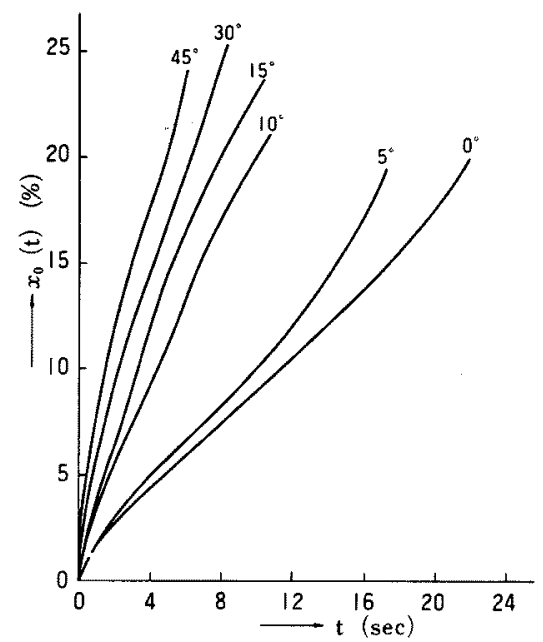

Fig. 14 Fabric elongation of different bias cut fabrics tested under same ramp load

which compared the load $f_{i}(t)$ tested under the same ramp load input on various bias cut angles (based on the warp direction). Here, the velocity error could be eliminated completely as stated in (5.1.A) in the range $K_{p f}=0.05-0.3$ $(\mathrm{cm} / \mathrm{kg}$ ), and Fig. 13 shows the characteristics of this tester in itself cleary. Fig. 14 shows the deformation of the test piece tested under the same ramp load input $(0.8 \mathrm{~kg} / \mathrm{sec})$. It is seen that the deformation of fabrics becomes large when the bias angle exceeds $5^{\circ}$.

\section{B. Deformation ${ }_{A}^{\text {weft }}$ yarn density}

The mechanical characteristics of fabrics are varied not only by the kinds of composed materials but also by the structure of fabrics. As an example the influence of the weft yarn density on the deformation properties in warp direction has been studied and compared for the same ramp load input $(0.23 \mathrm{~kg} / \mathrm{sec})$ in the following three cases.

a) weft yarn is completely avoided.

b) weft yarn is reduced to $1 / 3$ of the original.

c) original fabric (a control).

98

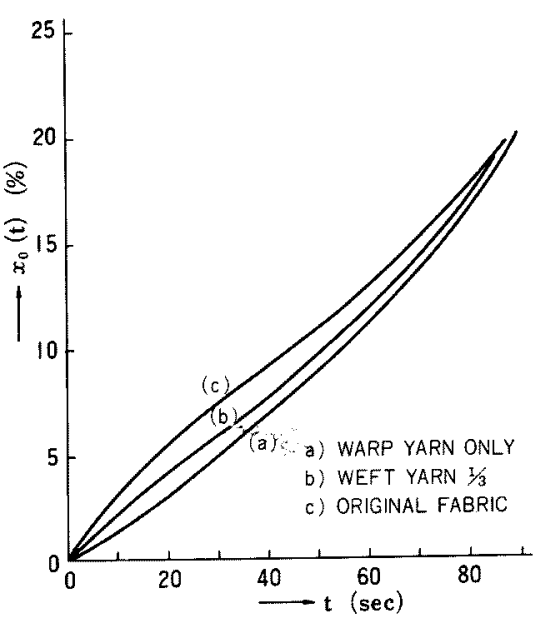

Fig. 15 Fabric elongation of various weft density tested under same ramp load input

Fig. 15 shows the results obtained for the basket weave $\left(\frac{3}{3}\right)$ fabric. a) is similar to the characteristics of the fiber tested under the ramp load input. c) the process of increasing deformation by the weft squeeze effect during extention b) is the medium of a) and c), showing the weft influence on the fabric deformation.

C. Frequency characteristics of load input

So far, the repeated load test of fabrics has not been done almost. Here, we show an example tested with the same test pieces as used in the preceding experiment. The fabric deformation under various sine wave load has been tested with $1 \mathrm{~kg}$ initial tention. Fig. 16 is the Bode diagram of this test, showing the relation between the output deformation $x_{o}(t)$ of the test piece and the actual load $f_{i}(t)$ imposed on a test piece. Judging from the ability of this tester and experimental results, the purpose of this investigation is attained at least. However, it is still left to study the relation be-

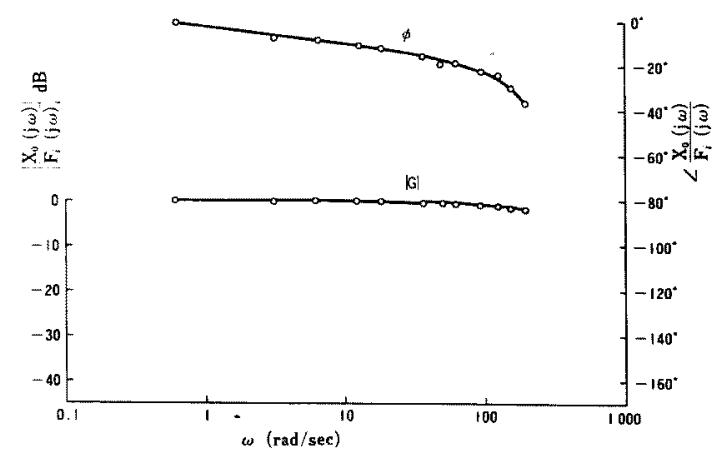

Fig. 16 Load input frequency response of cotton plain weave fabric

Journal of The Textile Machinery Society of Japan 
tween the hunting of the hydraulic servo system and the mechanism of load detector and its gain constant.

\section{Conclusions}

Assuming that the fabric deformation follows Voigt's model, a load input tensile tester was made, the performance of which should not be influenced by the mechanical properties of the material to be tested, and its ability was studied experimentally.

Especially we discussed the conditions to avoid the velocity error of the tester, to eliminate the influence of load on the hydraulic servo system, and to insert the compensation circuit in order to make the performance of the tester independent of the properties of the test material. Thus it was made clear that the hydraulic servo system of our tester must be composed of an integral element.

This tester can be applied to various load inputs which simulate the actual production process. Accordingly, our tester is useful for testing mechanical properties of fabrics hitherto difficult to examine, will be successfully used to test mechanical properties of general industrial materials by using different springs which convert the displacement to tensile load.

\section{Appendix}

In Fig. 4, a transfer function $G_{6 f}(s)$, from the deflection voltage $\varepsilon(t)$ to the load $f_{i}(t)$, is

$$
G_{\imath f}(s)=\frac{T_{2} s+1}{T_{1} s+1} \cdot \frac{K_{m}}{s} \cdot \frac{K_{s}\left(T_{p f} s+1\right) /\left(1+K_{s} K_{p f}\right)}{T_{p f} s /\left(1+K_{s} K_{p f}\right)+1}
$$

Here, putting

$$
T_{2}=\frac{T_{p f}}{1+K_{s} K_{p f}}
$$

and exchanging variables as follows, the controllability and the observability of this control system are as follows:

$$
\begin{aligned}
& A=T_{2}=\frac{T_{p f}}{1+K_{s} K_{p f}}, \quad B=1 \\
& C=\frac{K_{s} T_{p f}}{1+K_{s} K_{p f}}, \quad D=\frac{K_{s}}{1+K_{s} K_{p f}} \\
& E=1 / K_{m}, \quad F=T_{1}, \quad G=1, \\
& G_{s f}(s)=\frac{y(s)}{u(s)}=\frac{A s+B}{F s+G} \cdot \frac{C s+D}{E s(A s+B)}
\end{aligned}
$$$$
\text { and }
$$

From the above transfer function, the following state equations and the output equations are induced.

$$
\begin{aligned}
& \dot{X}=A X+B u \\
& y=C X \\
& \begin{array}{l}
X=\left(\begin{array}{l}
x_{1} \\
x_{2} \\
x_{3}
\end{array}\right), \quad A=\left(\begin{array}{ccc}
-G / F & 0 & 0 \\
0 & 0 & 1 \\
1 / E & 0 & -B / A
\end{array}\right) \\
B=\left(\begin{array}{c}
\frac{1}{F}\left(B-\frac{A G}{F}\right) \\
0 \\
\frac{A}{E F}
\end{array}\right), \quad C=\frac{A}{E}(0, D, C)
\end{array}
\end{aligned}
$$$$
\text { where }
$$

Vol. 20 No. 4 (1974)
From the above description, the signal flow diagram is shown as Fig. (A)

Signal flow diagram (A)

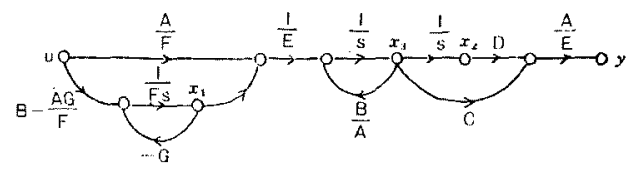

By the following nonsigular matrix and canonical state variable:

$$
\begin{aligned}
& \boldsymbol{X}=\boldsymbol{P} \boldsymbol{X}^{*} \\
& \boldsymbol{P}=\left(\begin{array}{ccc}
0 & 1 & 0 \\
1 & -\frac{A F^{2}}{G E(B F-A G)} & -\frac{A}{B} \\
0 & \frac{A F}{E(B F-A G)} & 1
\end{array}\right)
\end{aligned}
$$

and

$$
\begin{aligned}
& \alpha=-\frac{A F^{2}}{G E(B F-A \bar{G})}, \quad \beta=\frac{A F}{E(B F-A G)} \\
& \gamma=-\frac{A}{B},
\end{aligned}
$$

we have the state equations and the output equations of $X^{*}$ as follows.:

$$
\begin{aligned}
\boldsymbol{A}^{*}=\boldsymbol{P}^{-1} \boldsymbol{A} \boldsymbol{P} & =-\left(\begin{array}{ccc}
\alpha-\beta \gamma, & -1 & \gamma \\
-1 & 0 & 0 \\
\beta & 0 & -1
\end{array}\right) \boldsymbol{A}\left(\begin{array}{ccc}
0 & 1 & 0 \\
1 & \alpha & \gamma \\
0 & \beta & 1
\end{array}\right) \\
& =-\left(\begin{array}{ccc}
0 & -\frac{G}{F}(\alpha-\beta \gamma)+\gamma\left(\frac{1}{E}-\frac{B \beta}{A}\right)-\beta, & -1-\frac{B}{A} \gamma \\
0 & G / F & 0 \\
0 & -\frac{G}{F} \beta-\frac{1}{E}+\frac{B \beta}{A} & \frac{B}{A}
\end{array}\right) \\
& =\left(\begin{array}{ccc}
0 & 0 & 0 \\
0 & -G / F & 0 \\
0 & 0 & -B / A
\end{array}\right) \\
\boldsymbol{B}^{*} & =\boldsymbol{P}^{-1} B=\left(\begin{array}{cc}
\frac{A}{E G} \\
\frac{B F-A G}{F-} \\
0
\end{array}\right) \\
C^{*} & =C P=A / E(0, D, C) P \\
& =\frac{A}{E}\left(D, \frac{A F(C G-D F)}{E G(B F-A G)}, \frac{C B-A D}{B}\right)
\end{aligned}
$$

From the above description, the state equation and the output equation expressed in canonical form are as follows:

$$
\begin{aligned}
& \dot{X}^{*}=A^{*} X^{*}+B^{*} u \\
& y=C^{*} X^{*}
\end{aligned}
$$

These are expressed by the following signal flow diagram (B). 
Signal flow diagram (B)

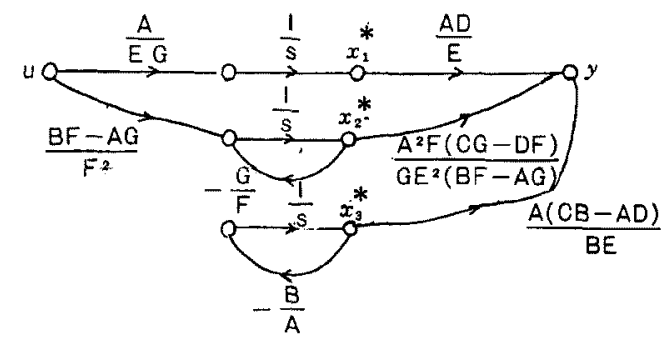

From eq. (A-10), the observability of this system is:

$$
\begin{aligned}
& \operatorname{det}\left(B^{*}, A^{*} B^{*}, A^{* 2} B\right) \\
= & \operatorname{det}\left(\begin{array}{ccc}
A / E G & 0 & 0 \\
\frac{B F-A G}{F^{2}}, & -\frac{G(B F-A G)}{F^{3}}, & \left(\frac{G}{F^{2}}\right)^{2}(B F-A G) \\
0 & 0 & 0
\end{array}\right)
\end{aligned}
$$$$
=0
$$

Here, it becomes equal zero for any A, B, E, F, G. Accordingly, it is not observable. This is clear from the signal flow diagram shown in Fig. (2). Further it becomes observable when the following relation is satisfied:

$$
\operatorname{det}\left(\begin{array}{ccc}
A D / E & 0 & 0 \\
\frac{A^{2} F(C G-D F)}{E^{2} G(B F-A G)}, & -\left(\frac{A}{E}\right)^{2} \frac{C G-D F}{B F-A G}, & \frac{A^{2} G(C G-D F)}{E^{2} F(B F-A G)} \\
\frac{A(C B-A D)}{B E}, & -\frac{C B-A D}{E}, & \frac{B(C B-A D)}{A E}
\end{array}\right)
$$$$
\neq 0
$$

\section{Reference}

[1] T. E. Olofson: Ann. Sci. Text. Belges, p. 7-30 (1955)

[2] R. Meredith and J. W. Shearle: Physical Method of Investigating Textiles, p. 227, Textile Book Publisher (1958)

[3] P. B. Checkland, T. H. Bull, E. J.Bakku: Text. Res. J., 28, 399 (1958)

[4] W. F. Kilby: J. Text. Inst., 54, T 9 (1963)

[5] W. D. Freston, Jr. M. M. Platt and M. M. Schoppee: Text. Res. J., 37, 948 (1967)

[6] M. Niwa, S. Kawabata, Y. Nanashima and H. Kawai: J. Tex. Mach. Soc. Japan, 23, 6, T123 (1970)

[7] T. Murakami: J. Japan Hydr. and Pne. Soc. 4, 1, 15 (1973)

[8] T. Murakami: J. Japan Hydr. and Pne. Soc. 4, 1, 17 (1973)

[9] F. R. Eirich: Rheology, Vol. V, Theory and Applications, p. 523, Academic Press (1969)

[10] F. Murakami: Process analysis for textile engineers, 17, RIKO, Japan (1964)

[11] T. Murakami: J. Japan Hydr. and Pne. Soc. 4, 1, 16 (1973)

[12] Hydraulic servo technics: Edited by planning committee of the lecture note of hydraulic servo technics 6, 14 (1972)

[13] Handbook of Fibers: Edited by J. Fib. Sci. Tech. Japan, 210 (1968)

[14] same to (7)

[15] same to (11)

[16] same to (7) 